\title{
Reflexões sobre questões morais na relação de indígenas com os serviços de saúde
}

Ana Lucia de Moura Pontes ${ }^{1}$, Luiza Garnelo ${ }^{2}$, Sergio Rego ${ }^{3}$

\section{Resumo}

Os sistemas médicos são culturalmente moldados e podem ter impactos negativos naqueles que não compartilham essas bases. Discutimos a perspectiva indígena de questões morais na relação com os serviços de saúde na região do Alto Rio Negro/Amazonas/Brasil. Trata-se de pesquisa qualitativa que utilizou como técnicas a observação participante e entrevistas em duas comunidades na região do Alto Rio Negro. A transferência para serviços de saúde em área urbana foi identificada como a principal questão moral para os indígenas na região. A diversidade de tradições, culturas e valores dos povos indígenas influenciam na sua moralidade e tomada de decisões clínicas, que eram pouco compreendidas pelos profissionais de saúde. Na relação entre profissionais de saúde e usuários indígenas ficou evidente o choque entre habitus distintos e a configuração de uma relação entre estranhos morais. Essas condições dificultam o diálogo para a resolução de conflitos. Palavras-chave: Saúde de populações indígenas. Ética clínica. Bioética. Noroeste amazônico.

\section{Resumen}

\section{Reflexiones sobre cuestiones morales en la relación de indígenas con los servicios de salud}

Los sistemas médicos son culturalmente moldeados y pueden tener impactos negativos en los que no comparten estas bases. Se discute la perspectiva indígena de cuestiones morales en la relación con los servicios de salud en la región del Alto Rio Negro/Amazonas/Brasil. Fue un estudio cualitativo que utiliza las técnicas de la observación participante y entrevistas en dos comunidades de la región del Alto Rio Negro. La transferencia para servicios de salud en zona urbana fue identificada como la principal cuestión moral para los indios de la región. La diversidad de tradiciones, culturas y valores de los pueblos indígenas tienen influencia en su moralidad y la toma de decisiones clínicas que eran poco comprendidas por los profesionales de salud. En la relación entre los profesionales de salud y los usuarios indígenas se hizo evidente el choque entre los distintos habitus y la configuración de una relación entre extraños morales. Estas condiciones dificultan el diálogo para resolver los conflictos.

Palabras-clave: Salud de poblaciones indígenas. Ética clínica. Bioética. Noroeste del Amazonas.

\section{Abstract \\ Reflection on moral issues in the relation between indigenous people and health services}

Medical systems are culturally adapted and may have negative impacts on those that do not share these bases. We discussed the indigenous perspective on moral issues in the relation with health services in the region of Alto Rio Negro/Amazonas/Brazil. It was a qualitative research that used participant observation and interviews in two communities in the region of Alto Rio Negro as techniques. The transfer to health services in urban areas was identified as the main moral issue for the Indians in the region. The diversity of traditions, cultures and values of the indigenous people influence their morality and clinical decisions to be made, what used to be little understood by health professionals. In the relationship between health professionals and indigenous users it was clear the shock between different habits and the configuration of a relation between moral strangers. These facts hinder the dialogue to solve conflicts.

Key words: Health of indigenous people. Ethics, clinical. Bioethics. Northwest Amazon.

Aprovação Conep - CAAE 0058.0.031.000-11

1. Doutora analupontes@fiocruz.br Escola Politécnica de Saúde Joaquim Venâncio/Fiocruz, Rio de Janeiro/RJ, Brasil 2. Doutora luiza. garnelo@amazonia.fiocruz.br Instituto de Pesquisas Leônidas e Maria Deane/Fiocruz, Manaus/AM, Brasil 3. Doutor rego@ensp.fiocruz. br Escola Nacional de Saúde Pública Sergio Arouca/Fiocruz, Rio de Janeiro/RJ, Brasil. 
A percepção, compreensão e resolução dos problemas morais nos serviços de saúde são fortemente influenciadas pelo contexto, rural ou urbano, onde ocorrem ${ }^{1,2}$. Uma característica fundamental dos serviços de saúde em contextos rurais é que frequentemente as populações atendidas possuem conhecimentos, práticas e valores diferentes dos reconhecidos pelos profissionais de saúde. As variações de compreensões de práticas e de valores morais levam a episódios de conflitos nos serviços de saúde ${ }^{3}$. Turner ${ }^{4}$ afirma que nas últimas décadas vários estudos têm mostrado a considerável variação cultural nas formas como as famílias e os indivíduos entendem situações clínicas como o diagnóstico terminal, a definição de morte, o uso de alta tecnologia, entre outras.

As diferenças culturais e de tradições religiosas estão diretamente relacionadas com essa diversidade moral existente na sociedade. Portanto, na relação entre povos indígenas e profissionais de saúde essas diferenças deveriam ser tomadas como pressuposto. Ellerby e colaboradore ${ }^{3}$ destacam que as decisões éticas dos povos nativos diferem dos valores hegemônicos da sociedade ocidental. Ademais, o contexto histórico das relações de poder existentes entre povos indígenas e sociedades nacionais também influencia essa relação. No Brasil, durante séculos, agentes colonizatórios - como as instituições de saúde - desenvolveram o projeto de "civilizar" os indígenas, ou seja, entendiam que estes deveriam ser protegidos de si mesmos adotando os valores, costumes e habitus da cultura ocidental ${ }^{5}$. Assim, foram desconsiderados como sujeitos morais, com valores e conhecimentos próprios e capazes de tomar decisões.

A discussão sobre questões morais na relação de indígenas com os serviços de saúde é fundamental para a melhoria da qualidade do cuidado prestado a essa população, pois a falta de uma atenção culturalmente sensível pode ser responsável pelo não uso dos serviços de saúde e por seus maus resultados ${ }^{6,7}$. Em vários países é visível que a população indígena apresenta menor expectativa de vida e maiores prevalência e incidência de diversos agravos ${ }^{7-11}$. Os sistemas médicos são culturalmente moldados, no Brasil como em outros países, em conformidade com os valores ocidentais, cristãos e liberais, o que pode trazer impactos negativos àqueles que não compartilham essas bases ${ }^{2,7}$.

Turner ${ }^{4}$ destaca a relevância de estudos sobre o ponto de vista moral de membros de comunidades específicas. Sob tal enfoque, pretende-se, neste artigo, discutir a perspectiva indígena a respeito de questões morais na relação com os serviços de saúde na região do Alto Rio Negro/Amazonas/Brasil. Nossa análise partirá do reconhecimento de que profissionais de saúde e indígenas não compartiIham os mesmos habitus ${ }^{12,13} \mathrm{e}$, portanto, constituem-se como estranhos morais ${ }^{14}$. Nesse contexto, consideramos a ética discursiva como um caminho para a solução desses conflitos e forma de superar a relação colonizador-colonizado ${ }^{15}$.

\section{Habitus, estranhos morais e ética discursiva}

O habitus, segundo Bourdieu, são esquemas de disposições duradouras, adquiridos nos processos de socialização, que incluem dois componentes: os princípios ou valores que regulam a conduta cotidiana (ethos) e as posturas e disposições do corpo (hexis corporal) ${ }^{13,16}$. Portanto, indígenas e não indígenas, que não compartilham o mesmo mundo social, desenvolvem diferentes atitudes e maneiras de agir, perceber e refletir.

Os agentes portadores do mesmo habitus não precisam entrar em acordo para agir da mesma maneira ${ }^{16}$, pois a codificação comum assegura uma comunicação mínima ${ }^{13}$. Todavia, se as condições objetivas ou o contexto se modificam, pode ocorrer uma defasagem entre o habitus antigo e as novas condições, que levariam os agentes a atitudes consideradas inadequadas, desencadeando conflitos ${ }^{16}$.

Engelhardt Jr. ${ }^{14}$ denomina estranhos morais aqueles que não compartilham premissas, regras ou autoridades morais, incorporados nos habitus de indivíduos e grupos. Por conseguinte, os amigos morais seriam aqueles que compartilham uma moralidade continuísta em um grupo de pessoas. Para resolver as controvérsias, os amigos morais podem recorrer ao argumento moral ou a uma autoridade reconhecida, mas os estranhos morais não: teriam que resolver por meio de um acordo comum, pois não compartilham as mesmas regras, pressupostos ou autoridades morais. $O$ autor afirma que 0 não compartilhamento de uma moralidade essencial não significa a impossibilidade de compreensão da perspectiva do outro, de modo que propõe a resolução de conflitos por meio de acordos.

Concordamos com a perspectiva da ética discursiva, que tem sido trabalhada no contexto da relação entre indígenas e não indígenas no Brasil por Oliveira e Oliveira ${ }^{15}$ e Oliveira ${ }^{17}$ usando como fundamento o conceito de comunidade de comunicação e de argumentação de Karl-Otto Apel. A comunidade de comunicação é um espaço social marcado por relações dialógicas, mas que para funcionar democraticamente 
como comunidade de argumentação necessita de um acordo intersubjetivo das normas e regras entre os interlocutores ${ }^{15}$. Contudo, no contexto interétnico de uma sociedade dirigida por um Estado uniétnico, observa-se a hierarquização de uma cultura sobre a outra, de modo a comprometer as condições de simetria e igualdade necessárias para um diálogo efetivamente democrático. Este é o desafio que encontramos no contexto das relações entre profissionais de saúde e indígenas para a resolução de conflitos morais.

\section{Subsistema de saúde indígena no Brasil}

A partir de 1999 implantou-se um subsistema específico de atenção à saúde dos povos indígenas integrado ao sistema nacional de saúde brasileiro. A Política Nacional de Atenção à Saúde dos Povos Indígenas (Pnaspi) prevê a implantação de um modelo de atenção cuja operacionalização é baseada em 34 distritos sanitários especiais indígenas (Dsei), responsáveis por oferecer o acesso à atenção integral à saúde dos povos indígenas ${ }^{18}$. Os distritos oferecem serviços de atenção primária em saúde realizados por equipes multiprofissionais formadas por médicos, enfermeiros, odontólogos, técnicos de enfermagem e agentes indígenas de saúde (AIS) que devem atuar nas aldeias indígenas. Essa rede local articulase com o restante do sistema de saúde (SUS) para a oferta de serviços de média e alta complexidade.

Uma das diretrizes fundamentais desse subsistema é a atenção diferenciada, que preconiza a adequação dos serviços por meio da preparação dos profissionais de saúde para a atuação em contexto intercultural, a articulação com os sistemas tradicionais de saúde e a diferenciação das ações e tecnologias para os contextos locais. Porém, essa diretriz encontra muitas dificuldades para sua concretização nos distritos.

Alguns conflitos éticos oriundos da relação intercultural entre povos indígenas e sociedade nacional permanecem polêmicos, mas a literatura científica sobre as questões éticas que emergem com a implantação do subsistema de saúde indígena ainda é incipiente. A pouca bibliografia disponível foca a questão do infanticídio ${ }^{5,19-24}$ e a ética em pesquisa ${ }^{25}$.

\section{Método}

Esta é uma pesquisa qualitativa, interessada no "ponto de vista do ator" como produtor de sen- tidos ${ }^{26,27}$ e aprovada na Comissão Nacional de Ética em Pesquisa. As técnicas utilizadas para a coleta de dados foram a observação participante, na modalidade participante como observador ${ }^{28,29}$; o registro sistemático em caderno de campo; e revisão bibliográfica internacional.

O trabalho de campo foi realizado durante 40 dias consecutivos em duas comunidades do Baixo Rio Içana no Distrito Sanitário Especial Indígenas do Alto Rio Negro (Dseirn), situado no município de São Gabriel da Cachoeira Os principais informantes e tradutores foram quatro AIS - duas mulheres e dois homens - que atuavam nessas comunidades. Os nomes das comunidades e pessoas utilizados são fictícios.

A observação participante compreendeu o acompanhamento diário das atividades coletivas das comunidades, a prestação de cuidados pelos quatro AIS e os itinerários terapêuticos seguidos pelos doentes atendidos. Também foram realizadas conversas informais com moradores sobre suas experiências nos serviços de saúde nas cidades de São Gabriel da Cachoeira (SGC) e Manaus. Os registros no diário de campo foram revisados e categorizados, buscando identificar situações que, na perspectiva dos indígenas, representavam conflitos na relação com profissionais de saúde.

Também foi feita revisão bibliográfica no banco de dados do PubMed, em março de 2013, que serviu para a definição das categorias de análise e discussão dos dados empíricos.

\section{Contexto local do estudo}

O Dseirn está localizado no noroeste amazônico, região Alto Rio Negro, no município de São Gabriel da Cachoeira, na fronteira com a Colômbia. Atualmente, ali vivem 17 povos falantes de idiomas de três troncos linguísticos - Arawak, Tukano e Maku. Nosso estudo ocorreu na região do rio Içana, afluente do Rio Negro, onde vivem os membros da etnia Baniwa ${ }^{30}$, alvo de interesse da pesquisa.

O estudo foi realizado no baixo curso do Rio Içana, nas aldeias Baniwa, ficticiamente denominadas de Açaí e Buriti. A área do Baixo Içana compreende uma região geopolítica localizada entre a foz do Rio Içana e o médio curso do mesmo rio, local sob antiga influência do processo colonizatório; espaço preponderante de trocas comerciais estabelecidas no regime de extrativismo de produtos da selva, cuja influência econômica se faz sentir até meados do século XX. 
A relativa proximidade com a sede municipal de São Gabriel da Cachoeira favoreceu o intenso contato interétnico, a continuada atuação de missões religiosas e o maior contato com agências da sociedade nacional, o que acentuou as transformações no modo Baniwa de viver ${ }^{31}$. A organização e reprodução da vida social Baniwa é fortemente apoiada em um sistema de saberes míticos e na forma de organização de parentesco, fundamentais para a compreensão da origem, manutenção e classificação das doenças nessa sociedade ${ }^{32,33}$.

\section{Resultados e discussão}

Nas comunidades estudadas as famílias preferencialmente buscavam resolver seus problemas de saúde com recursos locais, que abrangiam: a) os cuidados domésticos/familiares, mediante uso de plantas medicinais e/ou dietas e/ou automedicação com remédios industrializados; b) a consulta a terapeutas tradicionais indígenas; $c$ ) o atendimento pelos AIS; d) menos frequentemente, a busca de assistência junto a enfermeiros e técnicos de enfermagem das unidades de apoio denominadas de polos-base.

Em geral, a população indígena usava de forma concomitante os diferentes recursos de saúde existentes no território; para eles não existiam conflitos ou contradições no uso de terapêuticas biomédicas e tradicionais, conjuntamente, caracterizando-se um pluralismo médico que é, segundo Menéndez ${ }^{34}$, típico das sociedades latino-americanas, nas quais os sujeitos e grupos sociais realizam constantemente articulações entre as variadas formas de atenção à saúde.

Os AIS, como membros das equipes multiprofissionais de saúde do Dseirn, realizavam a primeira avaliação e conduta dos usuários indígenas do subsistema de saúde nas aldeias, bem como deliberavam sobre as primeiras condutas. Se concluíssem que o caso era grave ou que não possuíam recursos locais para resolver o problema de saúde identificado, se comunicavam por radiofonia com a sede do Dseirn em busca de orientação sobre procedimentos ou para solicitar a remoção do doente para a cidade.

Na cidade de SGC localiza-se uma Casa de Saúde do Índio (Casai), unidade de apoio que oferecia hospedagem e cuidados de saúde aos indígenas encaminhados para atendimento na rede de referência da sede do município (hospital e ambulatório), gerenciada pelas secretarias estadual e municipal de saúde, respectivamente. Quando necessário, os doentes podiam ser encaminhados à Manaus - nesses casos, a Casai de Manaus hospedava os pacientes e gerenciava a continuidade do cuidado.

Durante o trabalho de campo foi identificado que a remoção para a cidade gerava conflitos entre as famílias indígenas e as equipes do Dseirn. De acordo com os AIS, frequentemente as pessoas desistiam da remoção ou se recusavam a ser deslocadas ou a permitir a transferência de familiares para a cidade. Na literatura, encontramos que esse tipo de situação é também comum em outras regiões. Stamp e colaboradores ${ }^{35}$ relatam que a transferência para serviços em áreas urbanas é uma experiência intimidadora para indígenas de áreas remotas na Austrália, e diz respeito ao receio do ambiente e de pessoas desconhecidas, às dificuldades e custos nos deslocamentos e alojamento, às inadequações culturais dos serviços e às dificuldades de comunicação relacionadas ao bilinguismo desses povos e, por vezes, à pouca fluência nos idiomas nacionais.

$\mathrm{Na}$ etapa de análise da pesquisa foram selecionados os registros no diário de campo relacionados com as situações de remoção, relatados pelos usuários indígenas ou acompanhados pela pesquisadora, que serviram de material empírico para a discussão sobre a perspectiva dos indígenas acerca dos conflitos com os serviços de saúde.

Barreiras nos serviços e discriminação nos serviços de saúde

"Durante uma visita com o AIS Rodrigo, ele comenta com as três familias reunidas que eu tinha perguntado as razões pelas quais as pessoas não gostam de ir para a cidade. O sr. Abreu diz que a Casai mistura muita gente, que o local é sujo e bagunçado, porque os funcionários não limpam o local e dizem que os indígenas é que deveriam limpar. Depois Dona Maria diz que a Casai de Manaus é pior que a de SGC, que lá não tem onde colocar a rede, que tem que dormir no chão" (Trecho do diário de campo da comunidade Açaí).

Muitos relatos deixavam claro que, para os indígenas, as condições da Casai eram inadequadas para a hospedagem de pacientes e familiares. Essas experiências negativas eram reforçadas por histórias semelhantes de outras famílias nas Casai e nos demais serviços de saúde - esse tipo de percepção negativa dos serviços de saúde foi também identificado na Austrália por Stamp e colaboradores ${ }^{35}$ e Aspin ${ }^{9}$.

Os indígenas demonstravam desconfiança na relação com os profissionais não indígenas e insegu- 
rança quando transferidos para os serviços urbanos, pois nestes se sentiam desconfortáveis, maltratados e submetidos a condições inadequadas. Dessa forma, a adesão às orientações e tratamentos ofertados era prejudicada e a remoção recusada pelos pacientes ou seus familiares, situação igualmente referida por outros autores ${ }^{6,7,9,35}$. Stamp e colaboradores ${ }^{35}$ destacam que a falta de informação disponível para os indígenas sobre o que esperar dos hospitais de referência agrava esse desconforto e consideramos que isso pode também ser um fator relevante na região do Rio Negro.

Esse tipo de situação foi classificado por Betancourt ${ }^{6}$ como barreiras estruturais no acesso aos serviços, pois se refere a dificuldades encontradas devido aos desenhos da estrutura física e de gestão dos serviços de saúde, como a hospedagem e manutenção dos espaços. Para o autor, esse tipo de barreiras também inclui a ausência de intérpretes, demora nos atendimentos, dificuldades na referência a serviços especializados, ausência de material educativo e informações culturalmente sensíveis ${ }^{6}$. Essas outras dificuldades no acesso nos serviços de saúde também são enfrentadas pelos indígenas brasileiros e mais uma vez podem ser compreendidas como a falta de respeito ao que os identifica e caracteriza naquela comunidade em particular.

Apesar de a legislação brasileira ${ }^{36}$ determinar o atendimento humanizado, acolhedor e livre de qualquer discriminação nos serviços, o funcionamento das instituições de saúde reflete valores e concepções existentes na sociedade. E percebemos que visões estereotipadas sobre os modos de vida dos indígenas levam à discriminação desses usuários nos serviços - o que é também constatado por outros autores ${ }^{7,9,37}$. O registro de que existem disparidades no acesso e na qualidade do atendimento nos serviços de saúde relacionadas ao perfil étnico é relatado em diversos estudos ${ }^{2,6,9}$, embora tal situação ainda seja pouco discutida na bibliografia brasileira.

\section{O desafio da diversidade e da pluralidade médica na atenção}

Segundo Betancourt ${ }^{6}$, o reconhecimento da necessidade de adequação, ou de competência cultural dos serviços, tem se tornado mais evidente nos últimos anos e no caso da atenção à saúde direcionada aos povos indígenas é mais evidente e necessária, posição compartilhada por vários autores ${ }^{7,3,2}$.

Para cada cultura, os sintomas, as classificações e as terapêuticas para os problemas de saúde variam.
No caso dos Baniwa, a origem e a classificação das doenças são feitas a partir de um sistema de saberes míticos relacionados com guerras entre famílias de heróis criadores ${ }^{38}$. Garnelo e Buchillet ${ }^{33}$ estabelecem a taxonomia das doenças dos povos indígenas rionegrinos, entre os quais os Baniwa, cujos fundamentos são: doenças relacionadas com a agressão de seresespíritos; com envenenamentos, uma forma de bruxaria que tem lugar central na cosmologia Baniwa; com conflitos de gênero que remetem às guerras ancestrais entre homens e mulheres; doenças provocadas por Yoópinai - seres-espíritos da floresta; e doenças provocadas pelo contato, mas inseridas na cosmologia tradicional.

Tais especificidades da compreensão do processo saúde-doença coexistem com a oferta de serviços governamentais baseados em APS, que são utilizados pelos indígenas no enfrentamento de seus problemas de saúde. Porém, na região do Rio Negro os relatos dos indígenas revelaram o despreparo das instituições e dos profissionais de saúde para lidar com essas especificidades socioculturais do processo saúde-doença:

\section{"Pergunto por que as pessoas não gostam de ir para} SGC? A agente Denise diz que o problema era que na Casai a comida é ruim e eles misturam as etnias. Denise também fala que havia um senhor que começou com inchaço generalizado, ela pediu resgate e a família, a princípio, concordou, mas depois desistiu. A família achava que era doença tradicional (sopro) e queria tratar com remédio tradicional" (Trecho do diário de campo da comunidade Açaí).

Nesse relato identificamos algumas barreiras, classificadas como clínicas para Betancourt ${ }^{6}$, relacionadas às diferenças socioculturais no processo saúde-doença-cura e que levam a conflitos nos serviços. A incompreensão dessas diferenças culturais, por parte de profissionais de saúde e gestores, pode ser expressa, por exemplo, na inadequação cultural quando da organização de serviços oferecidos na Casai, tais como: a mistura de pessoas de diferentes etnias, entendida como uma situação de risco à saúde; a desconsideração das dietas e restrições alimentares tradicionais das famílias enfermas; e a proibição de especialistas, conhecedores de plantas, pajés e benzedores realizarem seus tratamentos nos espaços dos serviços públicos, além do não reconhecimento das doenças tradicionais.

Garnelo e Wright ${ }^{32}$ destacam que o deslocamento de doentes para a cidade e a permanência na Casai são eventos temidos para membros dessa etnia, pois a produção de alimentos por pessoas de 
outros grupos é identificada como situação de risco para adoecimento, não por razões biomédicas, mas por ameaçar o modo de vida. Em paralelo, mesmo entre os indígenas urbanizados existe o receio de não poder seguir a dieta adequada para cada fase vital, segundo as tradições. A internação em hospitais ou permanência na Casai gera a ruptura de regras dietéticas, bastante restritivas para os enfermos e suas famílias, que alguns grupos mantêm. Na maior parte dos casos, essas regras não são exclusivas para o indivíduo enfermo e se estendem à família, o que é diferente da lógica dos serviços de saúde, cujo foco de atenção é exclusivamente o enfermo. Além disso, para essas sociedades a presença da família serve de apoio para os doentes, característica encontrada em outros contextos ${ }^{3,35}$.

Para os indígenas, existe o uso complementar, não conflitivo, de recursos biomédicos e tradicionais; mas para os profissionais de saúde é muito difícil aceitar essa coexistência. Lorenzo ${ }^{39}$ enfatiza o frequente etnocentismo na implantação das ações de saúde, baseado em uma visão de que o saber científico deve esclarecer e/ou validar o saber local ${ }^{39}$. Dessa forma, observamos que as práticas locais dos especialistas de cura são toleradas nos territórios indígenas, mas não em serviços de saúde.

Atualmente, a maioria dos profissionais de saúde que atuam nos distritos não recebeu nenhuma preparação para trabalhar no contexto indígena. Vários autores brasileiros identificam o desconhecimento dos profissionais não indígenas da história, culturas e sistemas médicos dos povos indígenas ${ }^{39-41}$, e confirmam que os profissionais de saúde têm dificuldades na compreensão do que seria a atenção diferenciada ${ }^{41,42}$. As ações de saúde implementadas nos distritos e na Casai são baseadas nos programas nacionais de saúde, sem adequações significativas nas tecnologias, sistemas de informação ou metas. Em decorrência, as concepções universalistas dos profissionais de saúde concretizam-se em um cuidado tecnicamente padronizado aos doentes, sem quaisquer considerações em relação às suas singularidades culturais.

Diferentes valores morais, formas de autonomia e os conflitos durante a tomada de decisão

Pelo relato dos usuários indígenas percebese que nos serviços de saúde eles são solicitados a tomar decisões com base em valores biomédicos. Entretanto, segundo Garvey e colaboradores ${ }^{2}$, na perspectiva indígena frequentemente o compromisso com a qualidade de vida é maior do que a busca da cura, assim como o desejo de receber um cui- dado proporcionado por indígenas, que envolva a cultura, a comunidade e a espiritualidade. Para Ellerby e colaboradores ${ }^{3}$, devido a uma imposição de valores ocidentais as instituições de saúde também podem ser associadas à cultura de colonização.

Para os indígenas, os parâmetros que orientam a tomada de decisão em situações clínicas não diferem daqueles aplicados nas demais situações da vida. As decisões éticas indígenas seriam frequentemente situacionais e altamente dependentes de valores e do contexto familiar e comunitário ${ }^{3}$. No contexto rionegrino, o ethos é fortemente orientado pela família (parentesco) e vida comunal. Para Ellerby e colaboradores ${ }^{3}$, a ética indígena é mais bem entendida como um processo relacional e, de fato, percebeu-se no trabalho de campo que para os indígenas era extremamente importante, na tomada de decisão, a qualidade da relação com os profissionais de saúde, principalmente com relação à confiança, amizade e empatia.

Também percebemos que para os indígenas e suas famílias do Alto Rio Negro os riscos e benefícios para o indivíduo podem ser relativizados diante dos interesses da família e da comunidade, e a presença da família na tomada de decisão clínica é fundamental. Concordamos com autores ${ }^{2,43}$ que apontam que a ênfase dominante na sociedade ocidental no respeito à autonomia - entendida como uma tomada de decisão individual, que prioriza o consentimento individual do enfermo - deveria ser substituída por concepções coletivistas. Ao invés de atribuir a unidade da decisão autônoma ao indivíduo, esta pode ser a família ou a comunidade, como identificado por Hanssen ${ }^{43}$.

Os profissionais de saúde deveriam ser capazes de reconhecer os diferentes modos de tomada de decisão, de noção de família e das formas de comunicação dos usuários indígenas ${ }^{2}$. Observouse essa dificuldade durante o trabalho de campo, quando um membro da comunidade sofreu uma paralisia. Dada a possibilidade de o indivíduo ter sofrido um acidente vascular cerebral, tentou-se convencê-lo em aceitar a remoção rápida via transporte do Dseirn. Porém, desde a primeira conversa o enfermo afirmou desejar deslocar-se por meios próprios, o que levaria muito mais tempo. Somente após dois dias de diálogo compreendeu-se e foi aceito que o enfermo queria gerenciar seu cuidado com os próprios recursos, de modo que pudesse, no percurso, parar em outra comunidade para encontrar com seu filho, realizar tratamento tradicional, ficar hospedado na casa de familiares na cidade e procurar o hospital autonomamente e não pelo Dseirn. 
Essa postura inicial reflete o paternalismo que marca a prática médica no Brasil, que enfatiza que o profissional deve atuar sempre no sentido de restaurar a saúde e prolongar a vida dos pacientes, mesmo sem sua aprovação e inclusive contra sua vontade ${ }^{44}$. Esse ethos, entendido como valor que regula a conduta cotidiana dos profissionais, é justificado pela ideia de que o saber técnico seria o único critério válido para garantir o melhor interesse do paciente ${ }^{44}$. Menéndez ${ }^{45}$ enfatiza que no processo de hegemonia da biomedicina construiu-se a situação de paciente subordinado ao conhecimento médico, postura incorporada no habitus profissional.

Porém, no Brasil, Barroso ${ }^{46}$ refere que a Constituição de 1988 é um marco na garantia dos direitos individuais, adotando a concepção de dignidade humana como a capacidade de ser autônomo. Esse jurista defende que prevaleceria legalmente no país a ideia de que os indivíduos, com base em seus valores e princípios, sejam religiosos ou culturais, podem decidir sobre suas vidas e seus tratamentos médicos, inclusive podendo abrir mão de um direito fundamental, como a vida.

Mas também é nesse ponto que observamos que indígenas e não indígenas são estranhos morais. Autores do campo da saúde indígena ${ }^{47}$ relatam que a oferta de cuidado à saúde é fortemente impactada pelo conflito entre os valores de igualdade e individualidade da sociedade ocidental e a organização das etnias das terras baixas sul-americanas, caracterizadas como sociedades de pessoas (e não de indivíduos) e marcadas pela tensão entre igualitarismo e hierarquia nos grupos de parentesco. Este é o caso específico das etnias do Alto Rio Negro.

A noção fundamental de liberdade individual de escolhas, que separa o indivíduo do meio sociocultural, entra em conflito com a noção de construção coletiva da pessoa desses grupos $25,43,47,48$. A noção de indivíduo é algo particular e histórico e, atualmente, é base das teorias científicas, políticas, éticas e do trabalho em saúde na sociedade ocidental, mas projetá-la para outras sociedades gera equívocos e conflitos.

\section{Estratégias de resolução de conflitos}

Ao considerar o referencial da ética discursiva, o grande desafio no campo da saúde indígena é a constituição de comunidades de argumentação ${ }^{15,17}$. Oliveira e Oliveira ${ }^{15}$ apontam três espaços sociais para o diálogo democrático e negociação: a "microesfera", referente ao âmbito da família e da comunidade; a "mesoesfera" situada nas relações com os
Estados nacionais; e a "macroesfera" das deliberações e ações de organismos internacionais.

Internacionalmente, existem disposições legais e marcos que defendem o discurso indígena e indigenista. Contudo, no âmbito da "microesfera" observou-se que as regras e valores morais diferiam entre indígenas e profissionais de saúde. Ademais, o pouco tempo para diálogo durante os atendimentos, o curto período de permanência das equipes nas comunidades, a falta de acompanhamento longitudinal dos pacientes e a alta rotatividade dos profissionais entram em conflito com a importância dada pelos indígenas à qualidade das relações humanas na tomada de decisão e no processo terapêutico ${ }^{3,43}$ e dificultam a constituição de comunidades de argumentação entre profissionais e indígenas - situação encontrada em outros distritos ${ }^{49,50}$.

Quando ocorria discordância com a indicação de transferência para a cidade, os principais responsáveis pelo diálogo e convencimento eram os AIS. O Dseirn adotou como estratégia solicitar aos que se recusam a remover os enfermos que assinassem um "termo de responsabilidade", cujo maior objetivo parece ser isentar o Dseirn das consequências da decisão. Essa estratégia é usada em outros distritos e, no nosso entendimento, dificulta o diálogo democrático e o entendimento das motivações sobre as recusas.

Alguns profissionais, com mais tempo e experiência, aprenderam a dialogar com lideranças e a realizar reuniões comunitárias para discutir seu planejamento e avaliação de atividades ou os problemas. Esse tipo de postura era bem vista pelos indígenas e auxiliava na resolução de conflitos. Tal iniciativa denota a importância de o profissional de saúde estabelecer diálogo e de, por meio deste, buscar o conhecimento sobre a realidade dos povos indígenas, e aponta para tentativas de constituição de comunidades de argumentação entre usuários e profissionais.

Durante a estadia nos serviços de saúde em área urbana, os indígenas também relataram dificuldades para garantir seus direitos e desejos com relação às condutas terapêuticas. Para o atendimento de especialistas indígenas e uso de medicação tradicional necessitavam retornar para as comunidades. Alguns terapeutas indígenas entrevistados relataram estratégias e tentativas de atenderem de forma desapercebida nos serviços.

É importante destacar que a regulação dessa relação entre serviços de saúde e usuários indígenas está no âmbito da "mesoesfera", da formulação 
e implementação Pnaspi e de seus mecanismos de participação e constituição de comunidades de argumentação. No contexto atual, identifica-se diferentes entraves para a constituição de espaços democráticos de diálogo, pois as instâncias locais e distritais de controle social não têm sido efetivas. No âmbito nacional, verifica-se a incorporação de lideranças indígenas substituindo a construção de espaços de diálogo representativos; na última Conferência Nacional de Saúde Indígena, em 2013, os gestores utilizaram diferentes estratégias para manter sua hegemonia na condução das deliberações - e observa-se diferentes ações governamentais e políticas que retrocedem nos direitos indígenas.

\section{Considerações finais}

A recusa dos indígenas no que diz respeito à transferência para os serviços de saúde em área urbana, identificada no Dseirn, revelou diversos elementos conflitivos da relação entre indígenas e profissionais de saúde. Em nossa análise, esses relatos não se referiam a recusa à atenção biomédica propriamente dita, mas às barreiras culturais inerentes à estrutura e proposta operacional dos serviços, bem como à desconsideração da perspectiva dos indígenas sobre o processo terapêutico.

Foi também possível verificar conflito entre prioridades sociais, rituais ou familiares dos grupos estudados, cuja importância não era percebida pelos profissionais de saúde e, por isso, se tornavam competitivas com a oferta dos cuidados de saúde.
Essa situação tem levado a experiências negativas e desrespeitosas nos serviços, a falhas de comunicação, à insatisfação, à baixa aderência aos tratamentos, à desconfiança na relação com os profissionais, entre outros problemas. Destacamos que alguns dos problemas identificados não são específicos para essa população.

A questão moral subjacente reside na não consideração da perspectiva do sujeito indígena no seu modo de conduzir a vida e o processo terapêutico. Não considerar neste caso significa não conhecer ou respeitar e isso expressa a persistência da perspectiva etnocêntrica, que advoga que o indígena ainda precisa ser protegido de si próprio.

Nas situações discutidas entre profissionais de saúde com usuários indígenas ficaram evidentes os choques entre habitus distintos e a configuração de uma relação entre estranhos morais. Ademais, percebe-se que as condições e as características do encontro entre profissionais de saúde e usuários indígenas dificultam a constituição de comunidades de argumentação para a resolução de discordâncias.

É necessário que os profissionais atentem para as percepções, expressões e valores de cada usuário indígena, e que flexibilizem suas práticas diante de cada contexto. A valorização da família durante o cuidado e a tomada de decisão, o uso de intérpretes durante o processo, o reconhecimento e o respeito dos conhecimentos e práticas tradicionais de saúde parecem fundamentais para consolidar a relação entre profissionais de saúde e indígenas nos serviços de saúde.

Artigo produzido no âmbito do Projeto Saúde e Condições de Vida de Povos Indígenas na Amazônia, Programa de Apoio a Núcleos de Excelência - Pronex/Fapeam/CNPq, Edital 003/2009.

\section{Referências}

1. Nelson W, Pomerantz A, Howard K, Bushy A. A proposed rural healthcare ethics agenda. J Med Ethics. 2007;33:36-9.

2. Garvey G, Towney P, McPhee JR, Little M, Kerridge IH. Is there an aboriginal bioethic? J Med Ethics. 2004;30:570-5.

3. Ellerby JH, McKenzie J, McKay S, Gariépy GJ, Kaufert JM. Bioethics for clinicians: 18. Aboriginal Cultures. CMAJ. 2000;163(7):845-50.

4. Turner L. Bioethics in a multicultural world: medicine and morality in pluralistic settings. Health Care Anal. 2003;11(2):99-117.

5. Garnelo L. Bioethics and indigenous worlds: where do we situate ourselves? Cad Saúde Pública. 2010;26(5):853-78.

6. Betancourt JR, Green AR, Carrollo JE, Ananeh-Firempong O. Defining cultural competence: a practical framework for addressing racial/ethnic disparities in health and health care. Public Health Rep. 2003;118:293-302.

7. Wexler L. Behavioral health services "Don't work for us": cultural incongruities in human service systems for Alaska native communities. Am J Community Psychol. 2011;47:157-69. 
8. Coimbra CEA, Santos RV. Saúde, minorias e desigualdade: algumas teias de inter-relações com ênfase nos povos indígenas no Brasil. Ciênc Saúde Coletiva. 2000;5(1):125-32.

9. Aspin C, Brown N, Jowsey T, Yen L, Leeder S. Strategic approaches to enhanced health service delivery for aboriginal and Torres Strait Islander people with chronic illness: a qualitative study. BMC Health Serv Res. 2012;12:143.

10. McMurray A. Culture-specific care for indigenous people: a primary health care perspective. Contemp Nurse. 2008;28:165-72.

11. Tookenay VF. Improving the health status of aboriginal people in Canada: new directions, new responsibilities. Can Med Assoc J. 1996;155(11):1.581-683.

12. Bourdieu P. O senso prático. Petrópolis: Vozes; 2011.

13. Bourdieu P. Coisas ditas. São Paulo: Brasiliense; 2004.

14. Engelhardt HT. Fundamentos da bioética. São Paulo: Loyola; 1998.

15. Oliveira RC, Oliveira LRC. Ensaios antropológicos sobre moral e ética. Rio de Janeiro: Tempo Brasileiro; 1996.

16. Bonnewitz P. Primeiras lições sobre a sociologia de P. Bourdieu. Petrópolis: Vozes; 2003.

17. Oliveira RC. O trabalho do antropólogo. Brasília: Paralelo 15/Editora Unesp; 2000.

18. Brasil. Ministério da Saúde. Fundação Nacional de Saúde. Política Nacional de Saúde. Política Nacional de Atenção à Saúde dos Povos Indígenas. Brasilia: Ministério da Saúde/Fundação Nacional de Saúde; 2002.

19. Feitosa SF, Garrafa V, Cornelli G, Carvalho SJ. Bioethics, culture and infanticide in brazilian indigenous communities: the Zuruahá case. Cad Saúde Pública. 2010;26(5):853-78.

20. Lorenzo CFG. Is an interethnic ethic possible? Reflections on indigenous infanticide. Cad Saúde Pública. 2010;26(5):853-78.

21. Schramm FR. The morality of infanticide at the crossroads between moral pluralism and human rights culture. Reflections on indigenous infanticide. Cad Saúde Pública. 2010;26(5):871-3.

22. Segato RL. Leaving behind cultural relativism to endorse historical pluralism. Cad Saúde Pública. 2010;26(5):875-6

23. Lidorio R. Uma visão antropológica sobre a prática do infanticídio indígena no Brasil. Ultimato [Internet]. 2007 (acesso jun. 2012);(309). Disponível: http://www.ultimato.com.br/revista/ artigos/309/uma-visao-antropologica-sobre-a-pratica-do-infanticidio-indigena-no-brasil

24. Adinolfi VT. Enfrentando o infanticídio: bioética, direitos humanos e qualidade de vida das crianças indígenas. Rede Mãos Dadas. [Internet]. 2012 (acesso jun. 2012). Disponível: http:// xa.yimg.com/kq/groups/20451959/578391691/name/enfrentando_infanticidio.pdf

25. Coimbra CEA, Santos RV. Ética e pesquisa biomédica em sociedades indígenas no Brasil. Cad Saúde Pública. 1996;12(3):417-22.

26. Flick U. Desenho da pesquisa qualitativa. Porto Alegre: Artmed; 2009.

27. Chizzotti A. Pesquisa qualitativa em ciências humanas e sociais. $3^{a}$ ed. Petrópolis: Vozes; 2010.

28. Cicourel A. Teoria e método em pesquisa de campo. In: Guimarães AZ, organizadora. Desvendando máscaras sociais. $2^{a}$ ed. Rio de Janeiro: Livraria Francisco Alves Editora; 1980. p. 87-121.

29. Foote-White W. Treinando a observação participante. In: Guimarães AZ, organizadora. Op cit. 1980. p 77-86.

30. Instituto Socioambiental. Federação das Organizações Indígenas do Rio Negro. Mapa-livro povos indígenas do Rio Negro: uma introdução à diversidade socioambiental do noroeste da Amazônia brasileira. São Gabriel da Cachoeira/São Paulo: ISA/Foirn; 2006.

31. Garnelo L. Poder, hierarquia e reciprocidade: saúde e harmonia entre os Baniwa do Alto Rio Negro. Rio de Janeiro: Editora Fiocruz; 2003.

32. Garnelo L, Wright R. Doença, cura e serviços de saúde: representações, práticas e demandas Baniwa. Cad Saúde Pública. 2001;17(2):273-84.

33. Garnelo L, Buchillet D. Taxonomias das doenças entre os índios Baniwa (Arawak) e Desana (Tukano Oriental) do alto Rio Negro (Brasil). Horiz Antropol. 2006;12(26):231-60.

34. Menéndez E. Intencionalidad, experiencia y función: la articulación de los saberes médicos. Revista Antropología Social. 2005;14:33-69.

35. Stamp G, Miller D, Coleman H, Milera A, Taylor J. 'They get a bit funny about going': transfer issues for rural and remote Australian aboriginal people. Rural Remote Health. 2006;6(536):1-8.

36. Brasil. Ministério da Saúde. Carta dos direitos dos usuários da saúde. Brasília: Ministério da Saúde; 2007.

37. Silva CD. Cotidiano, saúde e política: uma etnografia dos profissionais de saúde indígena. [tese]. Brasília: Universidade de Brasilia; 2010.

38. Garnelo L. Mito, história e representação social de doença sexualmente transmissível entre os Baniwa, noroeste amazônico. In: Nascimento DR, Carvalho DM, Marques RC, organizadores. Uma história brasileira das doenças. Rio de Janeiro: Mauad; 2006. p. 24-36.

39. Lorenzo CFG. Desafios para uma bioética clínica interétnica: reflexões a partir da política nacional de saúde. Rev. bioét. (Impr.). 2011;19(2):335.

40. Bruno PRA. Saberes na saúde indígena: estudo sobre processos políticos e pedagógicos relativos à formação de agentes de saúde Tikuna no Alto Solimões (AM), Brasil. [tese]. Rio de Janeiro: Instituto Oswaldo Cruz; 2008. 
41. Langdon EJ, Diehl EE. Participação e autonomia nos espaços interculturais de saúde indígena: reflexões a partir do sul do Brasil. Saúde Soc. 2007;16(2):19-36.

42. Langdon JE, Diehl EE, Wiik FB, Dias-Scopel RP. A participação dos agentes indígenas de saúde nos serviços de atenção à saúde: a experiência em Santa Catarina, Brasil. Cad Saúde Pública. 2006;22(12):2.637-46.

43. Hanssen I. From human ability to ethical principle: an intercultural perspective on autonomy. Med Health Care Philos. 2004;7:269-79.

44. Rego S, Palácios M, Siqueira-Batista R. Bioética para profissionais de saúde. Rio de Janeiro: Editora Fiocruz; 2009.

45. Menéndez E. Antropologia médica: orientaciones, desigualdades y transacciones. Cuadernos de la Casa Chata 179. México: Ciesas; 1990.

46. Barroso LR. Legitimidade da recusa de transfusão de sangue por testemunhas de Jeová: dignidade humana, liberdade religiosa e escolhas existenciais. In: Azevedo AV, Ligiera WR. Direitos do paciente. São Paulo: Saraiva; 2012. p. 343-82.

47. Coimbra C, Garnelo L. Questões de saúde reprodutiva da mulher indígena no Brasil. In: Monteiro $\mathrm{S}$, Sansone L, organizadores. Etnicidade na América Latina: um debate sobre raça, saúde e direitos reprodutivos. Rio de Janeiro: Fiocruz; 2004. p. 153-74.

48. Seeger A, DaMatta R, Viveiros de Castro EB. A construção da pessoa nas sociedades indígenas brasileiras. Boletim do Museu Nacional (Antropologia). 1979;32:2-19.

49. Novo MP. Os agentes indígenas de saúde do Alto Xingu. Brasília: Paralelo; 2010.

50. Kelly JA. State healthcare and Yanomami transformation: a symmetrical anthropology. Tucson: The University of Arizona Press; 2011.

\section{Participação dos autores}

Ana Lucia de Moura Pontes coletou os dados, analisou-os e elaborou o trabalho para o doutorado em Saúde Pública (Ensp/Fiocruz); Luiza Garnelo coorientou o estudo, tendo participado da análise dos dados e redação final do artigo; Sergio Rego orientou o estudo, tendo participado da análise dos dados e redação final do artigo.

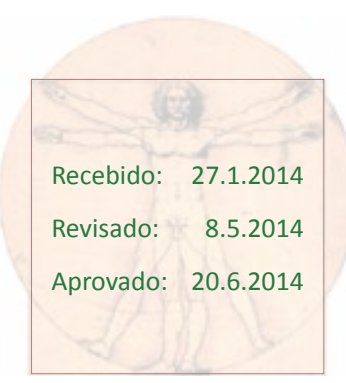

